\title{
Analyticity of free boundary in an optimal harvesting problem
}

\author{
Emamizadeh, B., Liu, Y.
}

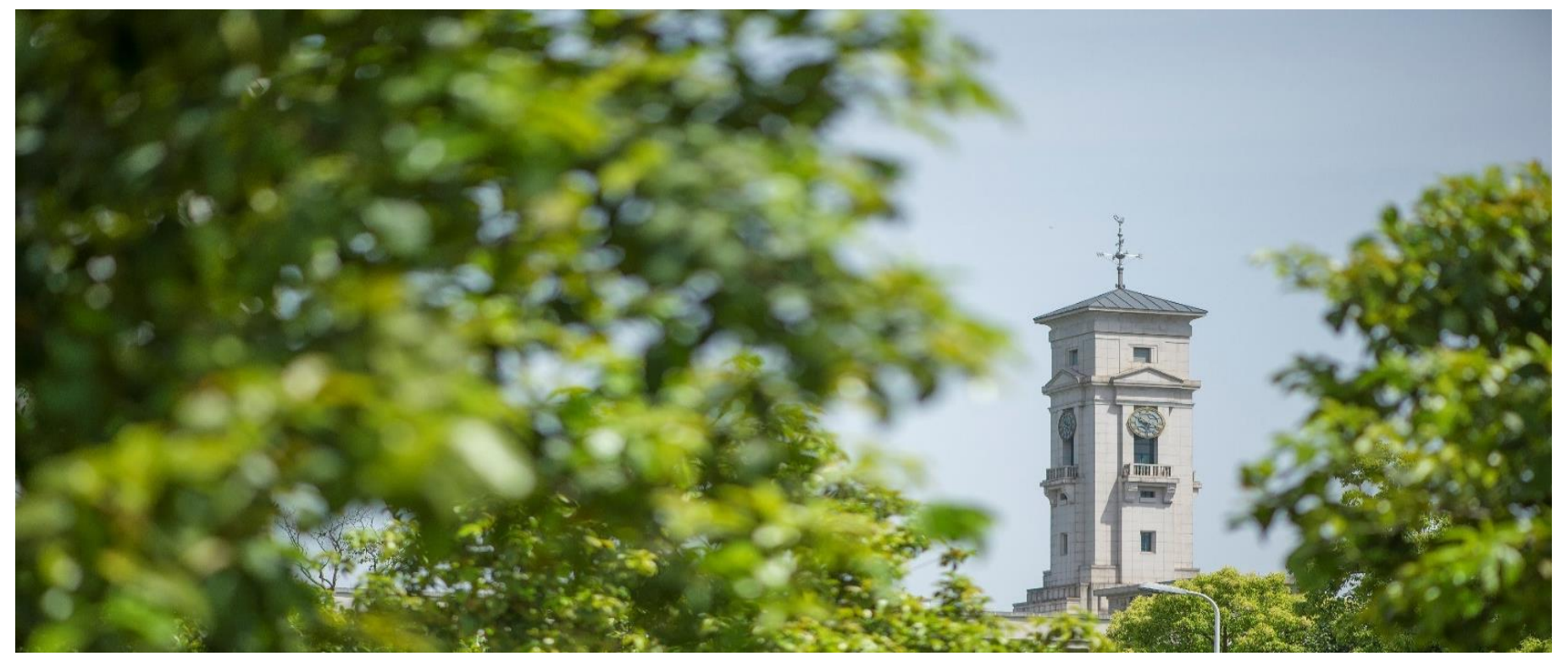


University of Nottingham Ningbo China, 199 Taikang East Road, Ningbo, 315100, Zhejiang, China.

First published 2020

This work is made available under the terms of the Creative Commons Attribution 4.0 International License:

http://creativecommons.org/licenses/by/4.0

The work is licenced to the University of Nottingham Ningbo China under the Global University Publication Licence:

https://www.nottingham.edu.cn/en/library/documents/researchsupport/global-university-publications-licence.pdf 


\title{
Analyticity of Free Boundary in an Optimal Harvesting Problem
}

\author{
Behrouz Emamizadeh, $\quad$ Yichen Liu ${ }^{\dagger}$
}

\begin{abstract}
This note revisits an optimisation problem pertaining to the optimal harvesting of a marine species. The existence of solutions and the corresponding optimal conditions they satisfy have already been proved. It is known that the optimal solutions can be identified with $n$-dimensional shapes. We will obtain an interesting result concerning the free boundary of the optimal shapes. Indeed, we will prove that if a parameter in the admissible set is kept sufficiently small then the free boundaries will be real analytic hypersurfaces.
\end{abstract}

Key Words: Free boundary, Harvesting, Semilinear equations, Regularity Mathematics Subject Classification: 35R35, 35J61, 92D40

\section{Introduction}

This note presents an interesting free boundary result that complements those in [12]. In this section we briefly review the essential parts of [12] that are relevant to our goal, and close the section with our main result.

In [12], the authors consider the following reaction-diffusion equation with logistic growth:

$$
\begin{cases}\frac{\partial u}{\partial t}=\varepsilon^{2} \Delta u+u-u^{2}+h(x, u) & \text { in } \Omega \times(0, \infty), \\ u(x, t)=0 & \text { on } \partial \Omega \times(0, \infty), \\ u(x, 0)=u_{0}(x) & \text { on } \Omega,\end{cases}
$$

*Behrouz.Emamizadeh@nottingham.edu.cn, School of Mathematical Sciences, University of Nottingham Ningbo China

†Yichen.Liu01@xjtlu.edu.cn, Department of Mathematical Sciences, Xi'an JiaotongLiverpool University, Suzhou, China 
where $\Omega \subseteq \mathbb{R}^{N}$ ( $N \geq 2$ ), a smooth ( $C^{2}$ is enough) bounded domain, denotes the inhabitant of a reproducing species (e.g. fish). The non-negative functions $u$ and $u_{0}$ stand for the population density and initial population of the species, respectively. The positive constant $\varepsilon^{2}$ is the diffusion constant, and $h(x, u)$ denotes the harvesting term which accounts for the human contributions to the system. Henceforth, we assume the harvesting is of constant type; i.e. $h(x, u)=-E(x) u$, where $E(x)$, a non-negative function, models the human efforts.

From the theory of parabolic equations, there exists a unique solution $u(x, t)$ of (1.1). The biological energy function associated with system (1.1) is given by:

$$
J_{\varepsilon}(u, E):=\frac{\varepsilon^{2}}{2} \int_{\Omega}|\nabla u|^{2} d x-\frac{1}{2} \int_{\Omega} u^{2} d x+\frac{1}{3} \int_{\Omega} u^{3} d x+\frac{1}{2} \int_{\Omega} E(x) u^{2} d x .
$$

It is well-known that there exists a function $u_{\infty}(x) \in H_{0}^{1}(\Omega)$ such that

$$
\lim _{t \rightarrow \infty}\left\|u(x, t)-u_{\infty}(x)\right\|_{H_{0}^{1}(\Omega)}=0,
$$

and satisfying the following steady state equation:

$$
\begin{cases}-\varepsilon^{2} \Delta u=u-u^{2}-E(x) u & \text { in } \Omega \\ u=0 & \text { on } \partial \Omega \\ u \geq 0 & \text { in } \Omega .\end{cases}
$$

For a suitable harvesting effort $E(x)$ and a small diffusion constant $\varepsilon^{2}$, we know the boundary value problem 1.3 has a unique positive solution, denoted $u_{E} \in H_{0}^{1}(\Omega)$, which coincides with $u_{\infty}$. By divergence theorem, $u_{E}$ satisfies the following integral identity:

$$
\varepsilon^{2} \int_{\Omega}\left|\nabla u_{E}\right|^{2} d x=\int_{\Omega} u_{E}^{2} d x-\int_{\Omega} u_{E}^{3} d x-\int_{\Omega} E(x) u_{E}^{2} d x .
$$

For details, we refer the readers to [2, 8, 12]. A natural question arises: What would be the best hunting strategy so that the species will not extinct and, at the same time, the residents near the inhabitant can benefit from this supply of food? As a start, we first observe that the strategy must manifest itself in the effort function $E(x)$, as this is the only human input into the system. Secondly, it is clear that $E(x)$ cannot be large across the inhabitant. Indeed if, for example, $E(x) \geq 1$, throughout $\Omega$, then (1.3) implies $u$ must be zero in $\Omega$, which implies the extinction of the species, a non-desirable situation. In [12], the following admissible set of strategies is considered:

$$
C_{\gamma, M}:=\left\{E(x) \in L^{\infty}(\Omega): 0 \leq E(x) \leq M, \int_{\Omega} E(x) d x=\gamma\right\},
$$


where $M>0$ and $0<\gamma<M|\Omega|$. Here, $M$ is the maximal harvesting effort. In the same paper, it is explained that the best strategy will be any solution to the following minimisation problem:

$$
\inf _{E \in C_{\gamma}} \Phi(E),
$$

where $\Phi(E):=J_{\varepsilon}\left(u_{E}, E\right)$. From the definition of $J_{\varepsilon}$ and (1.4) one easily finds the following "sign" result:

$$
\begin{aligned}
\Phi(E) & =\frac{\varepsilon^{2}}{2} \int_{\Omega}\left|\nabla u_{E}\right|^{2} d x-\frac{1}{2} \int_{\Omega} u_{E}^{2} d x+\frac{1}{3} \int_{\Omega} u_{E}^{3} d x+\frac{1}{2} \int_{\Omega} E(x) u_{E}^{2} d x \\
& =-\frac{1}{6} \int_{\Omega} u_{E}^{3} d x<0 .
\end{aligned}
$$

In a nutshell the significance of the above sign result is that numerous strategies can be ignored in the minimisation (1.5), if the diffusion constant is not sufficiently large. Indeed, when the diffusion constant fails to be large enough, the corresponding population $u_{E}$ will be identically zero, hence $J_{\varepsilon}(\cdot, E)$ will vanish at $u_{E}$. Thus, such a strategy cannot be optimal, according to the sign result.

It is shown in [12] (see also Theorem 2.5 below) that the optimal harvesting problem 1.5 has a solution in $C_{\gamma, M}$. Moreover, each minimiser $\hat{E}$ is of bang-bang type, i.e., a $\{0, M\}$-function, and the following optimality condition holds:

$$
\hat{E}=M \chi_{\left\{u_{\hat{E}}(x) \leq t\right\}} \text { for some } \quad 0<t<\max _{\Omega} u .
$$

Here, $\chi_{F}$ denotes the characteristic function over the set $F \subseteq \mathbb{R}^{N}$. The above optimality result captures two important features. Firstly, there is a region in which no harvesting should be implemented. In biology such a region is called the reserve marine zone, and the existence of such regions has always been a controversial matter amongst biologists. Secondly, the optimal condition asserts that in the harvesting region the effort must be gauged at the maximum possible amount i.e. $M$. Moreover, this region contains a layer around the boundary of the inhabitant, because $u_{\hat{E}}$ is continuous and vanishes on the boundary. Henceforth, we use $D_{\hat{E}}$ to denote the region of maximal harvesting effort corresponding to the optimal strategy $\hat{E}$, i.e., $D_{\hat{E}}=\left\{u_{\hat{E}}(x) \leq t\right\}$.

In this paper, we are interested in the regularity of the free boundary $\partial D_{\hat{E}}$. We shall prove that if either $\gamma$ or $M$ in the definition of $C_{\gamma, M}$ is sufficiently small then the boundary of $D_{\hat{E}}$ within $\Omega$ (which is a free boundary) will be real analytic. The precise result is given in the following

Theorem 1.1. There is $\gamma_{0} \in(0,|\Omega|)$ such that for any $\gamma \in\left(0, \gamma_{0}\right)$, any $\varepsilon \in$ $\left(0, \varepsilon_{2}(\gamma)\right)$, and any optimal configuration $D_{\hat{E}}$ which corresponds to the optimal strategy $\hat{E}$, we have $\partial D_{\hat{E}} \cap \Omega$ is real analytic. 
In Theorem 1.1, the function $\varepsilon_{2}(\gamma)$ is defined in 2.3 below.

Remark 1.2. An immediate observation is that by scaling one can eliminate either $\gamma$ or $M$ in the definition of $C_{\gamma, M}$. We choose to eliminate $M$, and set

$$
C_{\gamma}:=\left\{E(x) \in L^{\infty}(\Omega): 0 \leq E(x) \leq 1, \int_{\Omega} E(x) d x=\gamma\right\} .
$$

Remark 1.3. The admissible set $C_{\gamma}$ has been used for a variety of optimisation and control problems, see for example [3, 5, 11]. The authors of the present paper have also used this set in rearrangement optimisation problems, where $C_{\gamma}$ is formulated in terms of a rearrangement class. Indeed, if we fix a measurable set $E_{0} \subseteq \Omega$ satisfying $\left|E_{0}\right|=\gamma$, then for the rearrangement class generated by $\chi_{E_{0}}$, denoted $\mathcal{R}$, the following holds

$$
\mathcal{R}=\left\{\chi_{E}:|E|=\gamma\right\} .
$$

It is a well known fact that $C_{\gamma}$ is the weak* closure (in $L^{\infty}(\Omega)$ ) of $\mathcal{R}$. Equivalently, $C_{\gamma}$ is the strong closure of the convex hull of $\mathcal{R}$ i.e. $\overline{c o(\mathcal{R})}$. Another known fact is that $\mathcal{R}$ is the extreme set of $\overline{c o(\mathcal{R})}$. In many rearrangement optimisation problems, where the admissible set is $\overline{c o(\mathcal{R})}$, it turns out that the optimal solutions can (and sometimes should be) selected from the extreme set, hence dramatically reducing the amount of work in relevant numerical simulations. For such optimisation problems the reader is referred to a sample of papers [6, 9, 13].

The paper is organised as follows: In Section 2, we will include all the useful results which assist us to prove Theorem 1.1; The last section is devoted to the proof of Theorem 1.1 .

\section{Preliminaries}

The boundary value problem (1.3) could only have the trivial solution; this will occur when the diffusion constant is large otherwise we are guaranteed to get a nontrivial solution $u_{E}$. These facts plus the variational formulation of $u_{E}$ are stated in the following lemma. For proofs see Proposition 2.1 in [12], and Lemma 8 in [8].

Lemma 2.1. Assume that $|\{x \in \Omega: E(x)<1\}|>0$. Then, there exists an $\varepsilon_{1}=$ $\varepsilon_{1}(E)>0$ such that

- When $\varepsilon \in\left[\varepsilon_{1}, \infty\right)$, the problem (1.3) has only the trivial solution $u_{E} \equiv 0$.

- When $\varepsilon \in\left(0, \varepsilon_{1}\right)$, the problem $(1.3)$ has a unique positive solution $u_{E} \in$ $C^{1, \alpha}(\bar{\Omega}) \cap H_{0}^{1}(\Omega)$ satisfying $0<u_{E}(x)<1$ for all $x \in \Omega$. Moreover, we have

$$
\Phi(E)=J_{\varepsilon}\left(u_{E}, E\right)=\inf _{v \in H_{0}^{1}(\Omega)^{+}} J_{\varepsilon}(v, E) .
$$


Regarding $\varepsilon_{1}(\cdot)$ the following remarks are in order; see [2, 7] for details.

Remark 2.2. The exact value of $\varepsilon_{1}(E)$, in Lemma 2.1, is $\lambda_{1}^{-1 / 2}$. Here $\lambda_{1}$ denotes the first positive eigenvalue of

$$
\begin{cases}-\Delta z=\lambda(1-E(x)) z & \text { in } \quad \Omega \\ z=0 & \text { on } \partial \Omega .\end{cases}
$$

Remark 2.3. $\varepsilon_{1}(\cdot)$ is decreasing in the sense that if $E_{1}(x) \geq E_{2}(x)$ a.e. in $\Omega$, then $\varepsilon_{1}\left(E_{1}\right) \leq \varepsilon_{1}\left(E_{2}\right)$.

The dependence of the diffusion constant on $E$ is troublesome. For this reason we define:

$$
\varepsilon_{2}(\gamma):=\sup _{E \in C_{\gamma}} \varepsilon_{1}(E)
$$

and state the following (see Proposition 2.3 in [12])

Lemma 2.4. The supremum in (2.3) is achieved.

We are now in a position to state the results concerning the solvability of (1.5) and the optimality condition satisfied by minimisers, see [8], or Theorem 2.4 in [12] for the proof.

Theorem 2.5. Given $\gamma>0$, for $\varepsilon \in\left(0, \varepsilon_{2}(\gamma)\right)$, the minimisation problem (1.5) is solvable. Moreover, for each minimiser $\hat{E} \in C_{\gamma}$, there exists a $t \in(0,1)$ such that

$$
\hat{E}=\chi_{\left\{u_{\hat{E}}(x) \leq t\right\}},
$$

where $u_{\hat{E}}$ is the unique positive solution corresponding to $\hat{E}$ as in Lemma 2.1 .

The following auxiliary boundary value problem shall be used to achieve our goals. It should be seen as the limit case (when $\gamma \rightarrow 0$ ) of (1.3):

$$
\begin{cases}-\varepsilon^{2} \Delta w=w-w^{2} & \text { in } \quad \Omega, \\ w=0 & \text { on } \partial \Omega, \\ w \geq 0 & \text { in } \Omega .\end{cases}
$$

Proposition 2.6. When $\varepsilon \in\left(0, \varepsilon_{1}(0)\right)$, the problem (2.4) has a unique positive solution $w \in C^{1, \alpha}(\bar{\Omega}) \cap H_{0}^{1}(\Omega) \cap C^{\infty}(\Omega)$ satisfying $0<w(x)<1$ for all $x \in \Omega$. Moreover, every level set of $w$ has measure zero, i.e.,

$$
\forall t \in(0,1): \quad|\{x \in \Omega: w(x)=t\}|=0 .
$$

Proof. Based on Lemma 2.1 and standard elliptic regularity results, we only need to verify the last assertion; namely, that the level sets have zero measure. To this end we invoke Lemma 7.7 in [10]; together with the fact that $0<w<1$, we infer the desired result. 
The following comparison result is standard, see [1], or Lemma 4.3 in [2], for a proof.

Lemma 2.7. Let $m \in L^{\infty}(\Omega)$. Suppose that $\underline{u}, \bar{u} \in H^{1}(\Omega) \cap C(\bar{\Omega})$ satisfy $\underline{u} \leq \bar{u}$ in $\Omega$, and (in the weak sense)

$$
\begin{aligned}
& \begin{cases}-\varepsilon^{2} \Delta \underline{u} \leq m(x) \underline{u}-\underline{u}^{2} & \text { in } \quad \Omega, \\
\underline{u} \leq 0 & \text { on } \quad \partial \Omega,\end{cases} \\
& \begin{cases}-\varepsilon^{2} \Delta \bar{u} \geq m(x) \bar{u}-\bar{u}^{2} & \text { in } \Omega, \\
\bar{u} \geq 0 & \text { on } \partial \Omega,\end{cases}
\end{aligned}
$$

Then, the following boundary value problem

$$
\begin{cases}-\varepsilon^{2} \Delta u=m(x) u-u^{2} & \text { in } \quad \Omega, \\ u=0 & \text { on } \quad \partial \Omega\end{cases}
$$

has a solution $u \in H_{0}^{1}(\Omega)$ satisfying $\underline{u} \leq u \leq \bar{u}$.

An immediate consequence of Lemma 2.7 is the following:

Corollary 2.8. Assume that $|\{x \in \Omega: E(x)<1\}|>0$, and let $\varepsilon \in\left(0, \varepsilon_{1}(E)\right)$. Suppose $w, u$ are unique positive solutions of (2.4) and (1.3) respectively. Then, $0<u \leq w<1$ in $\Omega$.

Proof. We set $m=\bar{u} \equiv 1$ and $\underline{u}=u$. By Proposition 2.6, $w$ is the unique positive solution of 2.4 that satisfies $0<w<1$ in $\Omega$. Hence, Lemma 2.7 implies that $0<u \leq w<1$ in $\Omega$.

To prove Theorem 1.1 we shall use the following seemingly simple but powerful result from [4].

Lemma 2.9. Let $h(\xi)$ with $\xi \in \mathbb{R}$ be a locally bounded function. Let $v \in C^{1}(\Omega)$ satisfy (in the weak sense)

$$
\Delta v=h(v) .
$$

Assume at the point $x_{0} \in \Omega,\left|\nabla v\left(x_{0}\right)\right| \neq 0$. Then, there exists a ball $B, x_{0} \in B$, such that the set,

$$
\left\{x \in B: v(x)=v\left(x_{0}\right)\right\}
$$

is a real analytic hypersurface of $\mathbb{R}^{N}$. 


\section{Proof of the Main Result}

We begin by considering a particular case of 1.3 :

$$
\begin{cases}-\varepsilon^{2} \Delta u=u-u^{2}-\chi_{F}(x) u & \text { in } \Omega \\ u=0 & \text { on } \partial \Omega \\ u \geq 0 & \text { in } \Omega .\end{cases}
$$

Lemma 3.1. Let $F \subseteq \Omega$ with $|F|=\gamma$, and let $\varepsilon \in\left(0, \varepsilon_{1}\left(\chi_{F}\right)\right) \subseteq\left(0, \varepsilon_{1}(0)\right)$. Suppose $w, u$ are unique positive solutions of (2.4) and (3.1), respectively. Then, for every $p \in(1, \infty)$ and $\alpha \in[0,1)$, there is a positive constant $C=C(\Omega, \varepsilon, p, \alpha)$ such that

(i) $\|u-w\|_{W^{2, p}(\Omega)} \leq C\left(\gamma^{\frac{1}{3}}+\gamma^{\frac{1}{p}}\right)$;

(ii) $\|u-w\|_{C^{1, \alpha}(\bar{\Omega})} \leq C\left(\gamma^{\frac{1}{3}}+\gamma^{\frac{1-\alpha}{N}}\right)$.

Proof. Since $w \in H_{0}^{1}(\Omega)^{+}$, the minimality of $u$, see 2.1 , yields

$$
\begin{aligned}
& \frac{\varepsilon^{2}}{2} \int_{\Omega}|\nabla u|^{2} d x-\frac{1}{2} \int_{\Omega} u^{2} d x+\frac{1}{3} \int_{\Omega} u^{3} d x+\frac{1}{2} \int_{\Omega} \chi_{F} u^{2} d x=J_{\varepsilon}\left(u, \chi_{F}\right) \\
& \leq J_{\varepsilon}\left(w, \chi_{F}\right)=\frac{\varepsilon^{2}}{2} \int_{\Omega}|\nabla w|^{2} d x-\frac{1}{2} \int_{\Omega} w^{2} d x+\frac{1}{3} \int_{\Omega} w^{3} d x+\frac{1}{2} \int_{\Omega} \chi_{F} w^{2} d x .
\end{aligned}
$$

Adding the differential equation in (3.1) to the one in (2.4), multiplying the result by $u-w$, and integrating over $\Omega$, gives

$$
\begin{aligned}
& \varepsilon^{2} \int_{\Omega}|\nabla u|^{2} d x-\varepsilon^{2} \int_{\Omega}|\nabla w|^{2} d x= \\
& \quad \int_{\Omega} u^{2} d x-\int_{\Omega} w^{2} d x-\int_{\Omega}\left(u^{2}+w^{2}\right)(u-w) d x-\int_{\Omega} \chi_{F} u(u-w) d x .
\end{aligned}
$$

Substituting (3.3) into (3.2) yields

$$
\int_{\Omega}(w-u)^{3} d x \leq 3 \int_{\Omega} \chi_{F} w(w-u) d x \leq 3 \gamma,
$$

where in the last inequality we have used $0<u, w<1$ in $\Omega$. Next, by Corollary 2.8, $0<u \leq w<1$ in $\Omega$. So, (3.4) leads to

$\left\{\begin{array}{lll}\|u-w\|_{L^{p}(\Omega)} \leq|\Omega|^{\frac{3-p}{3}}\|u-w\|_{L^{3}(\Omega)} \leq C(\Omega, p) \gamma^{\frac{1}{3}} & \text { if } & p \in(1,3), \\ \|u-w\|_{L^{p}(\Omega)}=\left(\int_{\Omega}|u-w|^{3}|u-w|^{p-3} d x\right)^{\frac{1}{3}} \leq\|u-w\|_{L^{3}(\Omega)} \leq C \gamma^{\frac{1}{3}} & \text { if } & p \in[3, \infty),\end{array}\right.$ 
where we have used the Hölder's inequality and $0<u, w<1$ in $\Omega$. Subtracting (2.4) from (3.1), $u-w$ solves the following boundary value problem

$$
\begin{cases}-\varepsilon^{2} \Delta(Z)=Z(1-Z)-\chi_{F}(x) u & \text { in } \quad \Omega \\ Z=0 & \text { on } \quad \partial \Omega\end{cases}
$$

Applying Theorem 9.15 and Lemma 9.17, in [10], to [3.5) gives

$$
\|u-w\|_{W^{2, p}(\Omega)} \leq C(\Omega, \varepsilon, p) \gamma^{\frac{1}{3}}+C(\Omega, \varepsilon, p) \gamma^{\frac{1}{p}} \quad \text { for } \quad 1<p<\infty .
$$

Finally, the estimate (ii) follows from the Sobolev embedding $W^{2, p}(\Omega) \rightarrow C^{1, \alpha}(\bar{\Omega})$.

The following result is a direct consequence of the estimate (ii) in Lemma 3.1 .

Proposition 3.2. For any $\delta>0$, there is $\gamma_{0} \in(0,|\Omega|)$ such that for any $\gamma \in$ $\left(0, \gamma_{0}\right)$ and any $\varepsilon \in\left(0, \varepsilon_{2}(\gamma)\right)$, if we choose $t_{\gamma}$ satisfying $\left|\left\{w(x) \leq t_{\gamma}\right\}\right|=\gamma$ (which is possible due to Proposition 2.6) and $\chi_{\left\{u_{\hat{E}}(x) \leq t\right\}}$ is any minimiser of (1.5), then $\left|t_{\gamma}-t\right|<\delta$ and

$$
\left\{w(x) \leq t_{\gamma}-\delta\right\} \subseteq\left\{u_{\hat{E}}(x) \leq t\right\} \subseteq\left\{w(x) \leq t_{\gamma}+\delta\right\} .
$$

Proof. From the estimate (ii) in Lemma 3.1, we infer the existence of $\gamma_{0} \in(0,|\Omega|)$ such that $\left\|u_{\hat{E}}-w\right\|_{C(\bar{\Omega})}<\frac{\delta}{2}$. Let us set $[\Omega]^{s}=\{w(x) \leq s\}$ for $s \in(0,1)$, and $D_{\hat{E}}=\left\{u_{\hat{E}}(x) \leq t\right\}$. If $x \in D_{\hat{E}}$, then $x \in[\Omega]^{t+\frac{\delta}{2}}$ i.e. $w(x) \leq t+\frac{\delta}{2}$. So, $D_{\hat{E}} \subseteq[\Omega]^{t+\frac{\delta}{2}}$. Similarly, we have $[\Omega]^{t-\frac{\delta}{2}} \subseteq D_{\hat{E}}$. Thus we obtain

$$
[\Omega]^{t-\frac{\delta}{2}} \subseteq D_{\hat{E}} \subseteq[\Omega]^{t+\frac{\delta}{2}} .
$$

On the other hand, the function $f(r)=\left|[\Omega]^{r}\right|$ is continuous and increasing on $(0,1)$ since level sets of $w$ have zero measure (see Proposition 2.6). Recalling that $\left|D_{\hat{E}}\right|=\gamma$, we infer $\left|t_{\gamma}-t\right|<\frac{\delta}{2}$. It then follows $[\Omega]^{t_{\gamma}-\delta} \subseteq D_{\hat{E}} \subseteq[\Omega]^{t_{\gamma}+\delta}$, as desired.

Proof of Theorem 1.1 First, let us focus on the boundary value problem (2.4). As $\partial \Omega$ is $C^{2}$, we infer from Hopf's boundary point lemma, see for example, Lemma 3.4 in [10], that $|\nabla w(x)|>0$ on $\partial \Omega$. Since $w \in C^{1, \alpha}(\bar{\Omega})$, there exists $t_{w} \in(0,1)$ such that $|\nabla w(x)|>0$ on $\left\{w(x) \leq t_{w}\right\}$.

Let us set $\beta=\left|\left\{w(x) \leq \frac{t_{w}}{3}\right\}\right|<|\Omega|$ and $\delta=\frac{t_{w}}{3}$. We then infer from Proposition 3.2 the existence of $\gamma_{0} \in(0, \beta)$ such that for all $\gamma \in\left(0, \gamma_{0}\right)$, the following holds

$$
\left|t_{\gamma}-t\right|<\delta=\frac{t_{w}}{3} \quad \text { and } \quad\left\{u_{\hat{E}}(x) \leq t\right\} \subseteq\left\{w(x) \leq t_{\gamma}+\delta\right\} .
$$


Recalling that $\gamma<\gamma_{0}<\beta=\left|\left\{w(x) \leq \frac{t_{w}}{3}\right\}\right|$, it then follows that $t_{\gamma}<\frac{t_{w}}{3}$. Hence, $t_{\gamma}+\delta<\frac{2}{3} t_{w}$ and $C:=\min _{x \in A}|\nabla w(x)|>0$ with $A:=\left\{w(x) \leq \frac{2}{3} t_{w}\right\}$. So, we may use the estimate (ii) in Lemma 3.1 to further decrease $\gamma_{0}$, if necessary, to ensure $\left\|u_{E_{\gamma}}-w\right\|_{C^{1}(\bar{\Omega})}<\frac{C}{2}$ for $\gamma \in\left(0, \gamma_{0}\right)$ (here, $E_{\gamma}$ is a minimiser corresponding to $\gamma$ ). Observing that the inclusion $\left\{u_{E_{\gamma}} \leq t\right\} \subseteq A$ will still hold if $\gamma$ decreases, we then must have $\left|\nabla u_{E_{\gamma}}\right|>0$ on $\partial\left\{u_{E_{\gamma}}(x) \leq t\right\}$. To conclude, we use Lemma 2.9 as follows. Indeed, $u_{E_{\gamma}} \in C^{1, \alpha}(\bar{\Omega})$ satisfies

$$
\Delta v=h(v),
$$

where $h(\xi)=\frac{1}{\varepsilon^{2}} \chi_{G}(\xi) \xi-\frac{1}{\varepsilon^{2}} \xi(1-\xi) \in L_{l o c}^{\infty}(\mathbb{R})$, with $G=\{\xi: \xi \leq t\}$. Therefore, the assertion follows from Lemma 2.9 .

Conflict of interest. On behalf of all authors, the corresponding author states that there is no conflict of interest.

Acknowledgement. Y. Liu is supported by the XJTLU's research fund RDF-1702-22.

\section{References}

[1] H. Amann, Fixed point equations and nonlinear eigenvalue problems in ordered Banach spaces, SIAM Rev. 18 (1976), no. 4, 620-709.

[2] R. S. Cantrell and C. Cosner, Diffusive logistic equations with indefinite weights: population models in disrupted environments, Proc. Roy. Soc. Edinburgh Sect. A 112 (1989), no. 3-4, 293-318.

[3] S. Chanillo, D. Grieser, M. Imai, K. Kurata, and I. Ohnishi, Symmetry breaking and other phenomena in the optimization of eigenvalues for composite membranes, Comm. Math. Phys. 214 (2000), no. 2, 315-337.

[4] S. Chanillo, D. Grieser, and K. Kurata, The free boundary problem in the optimization of composite membranes, Differential geometric methods in the control of partial differential equations (Boulder, CO, 1999), Contemp. Math., vol. 268, American Mathematical Society, Providence, RI, 2000, pp. 61-81.

[5] S. J. Cox and J. R. McLaughlin, Extremal eigenvalue problems for composite membranes. I, II, Appl. Math. Optim. 22 (1990), no. 2, 153-167, 169-187.

[6] F. Cuccu, B. Emamizadeh, and G. Porru, Optimization problems for an elastic plate, J. Math. Phys. 47 (2006), no. 8, 082901, 1-12. 
[7] D. G. de Figueiredo, Positive solutions of semilinear elliptic problems, Differential equations (Sao Paulo, 1981), Lecture Notes in Math., vol. 957, Springer, Berlin-New York, 1982, pp. 34-87.

[8] B. Emamizadeh, A. Farjudian, and Y. Liu, Optimal harvesting strategy based on rearrangements of functions, Appl. Math. Comput. 320 (2018), 677-690.

[9] B. Emamizadeh and Y. Liu, Constrained and unconstrained rearrangement minimization problems related to the p-Laplace operator, Israel J. Math. 206 (2015), no. 1, 281-298.

[10] D. Gilbarg and N. S. Trudinger, Elliptic partial differential equations of second order, reprint ed., Classics in Mathematics, Springer-Verlag, Berlin, 2001.

[11] A. Henrot and H. Maillot, Optimization of the shape and the location of the actuators in an internal control problem, Boll. Unione Mat. Ital. Sez. B Artic. Ric. Mat. (8) 4 (2001), no. 3, 737-757.

[12] K. Kurata and J. Shi, Optimal spatial harvesting strategy and symmetrybreaking, Appl. Math. Optim. 58 (2008), no. 1, 89-110.

[13] Y. Liu and B. Emamizadeh, Converse symmetry and intermediate energy values in rearrangement optimization problems, SIAM J. Control Optim. 55 (2017), no. 3, 2088-2107. 